\title{
A Laser System for Detecting Surface Flaws of a Molding
}

\author{
Jing CHEN, Toshihiko YAMAGUCHI, Keiji TANIGUCHI*, Katsuhiko ASADA* \\ and Masahiro UEDA \\ Faculty of Education, Fukui University, Bunkyo 3-9-1, Fukui 910-0017 \\ *Department of Information Science, Faculty of Engineering, Fukui University, 3-9-1 Bunkyo, Fukui 910-0017
}

(Received May 7, 1998)

\begin{abstract}
An optical system for detecting the surface flaws of a molding, e.g., the brake disc of a car, has been proposed which involves a sheet-like laser beam of length, $l$, and width, $w$, as a probing light. Optimum conditions for the sheet like laser beam were found to be $l=10 \mathrm{~mm}$ and $w=0.5 \mathrm{~mm}$ for a flaw diameter of $2 \mathrm{~mm}$ which is the smallest diameter that can be detected in practice. The system consists of a sensor head including a light source and a light receiver, and a processing system including an amplifier, an A/D converter, a personal computer and a display. This system can detect all flaws in a few second by using a sampling frequency of data acquisition at $1 \mathrm{kHz}$.
\end{abstract}

Key Words: Laser system, Detection, Flaw, Molding

\section{Introduction}

Moldings have been used in many industrial fields. An example is the vehicular industry which includes the train and auto industries. The founded elements are usually cut and ground. Flaws on the cut surface produced by holes in the moldings can cause serious problems in some elements, particularly in brake disks. Many checks are needed to ensure that the moldings come up to standards. Checking for surface flaws is extremely important because braking a car to a stop is done on the surface itself. The detection of surface flaws has, heretofore, been carried out by the human eye. It is a very troublesome process and is timeconsuming work.

We have developed optical sensors for surface inspection that use light scattering on the surface, where the scattered light intensity depends on the state of the surface. These sensors have been successfully used for counting cloth filaments ${ }^{1)}$ and for measuring the roughness of a metal surface ${ }^{2)}$ and both scattered and reflected light intensities have been used for measuring the mean diameter of glass wool fibers. ${ }^{3)}$ Surface flaws can be detected by the same method. In this paper, we propose a method for detecting the surface flaws of a cut molding and analyse the system in terms of its speed, accuracy, and sensitivity for practical use.

\section{Method and System}

The underlying principle for this method is the same as the described in reference 3 , which is based on scattering and the reflection of laser light on the molding surface.

Figure 1 (a) shows the principle of the method and Fig.1 (b) the illustration of scattered and reflected light intensities. A sheetlike laser light illuminates a molding surface at about an angle of approximately $\theta=45^{\circ}$. A semiconductor laser of wavelength $\lambda=670 \mathrm{~nm}$ was used for the light source. Both scattered and reflected lights are received on a linear silicon photo-diode ar- ray about $10 \mathrm{~mm}$ in length. The photoreceiver consists of a silicon photodiode array with high sensitivity and a high-speed response. The scattered light receiver was fixed just above the flaw and the reflected light receiver was fixed at an angle of $\theta=$ $45^{\circ}$ as shown in Fig. 1 (a). The scattered light increases and the reflected light decreases when the laser light illuminates the flaw. Thus, the flaw will be detected.

Figure 2 shows the sensor head and a sample disc for this experiment. The brake disc was mounted on the turntable. Some artificial holes of various diameter were drilled on the disc surface to simulate flaws. Black paint was also applied on a small part of the surface to simulate a color change due to cutting.

Figure 3 shows the data processing system. Both scattered and reflected light intensities received on the photoreceiver were amplified, digitized by an 8 channel A/D converter, processed by a personal computer, and displayed on a monitor.

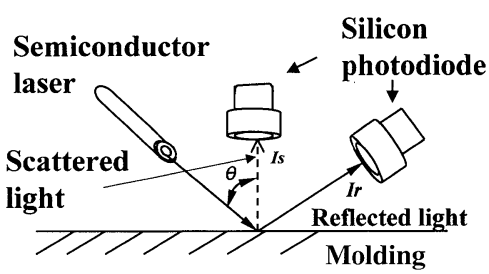

(a)

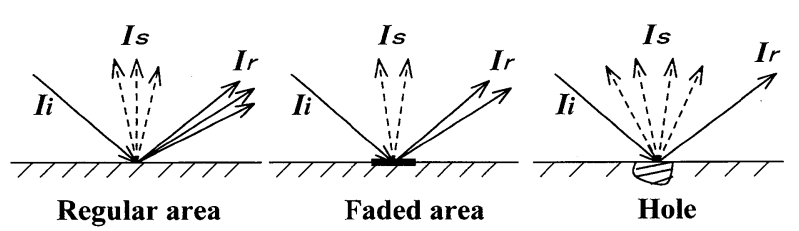

(b)
Fig.1 Principles of flaw detection. (a) Optical arrangement, (b) Illustration of scattered and reflected light intensities. 


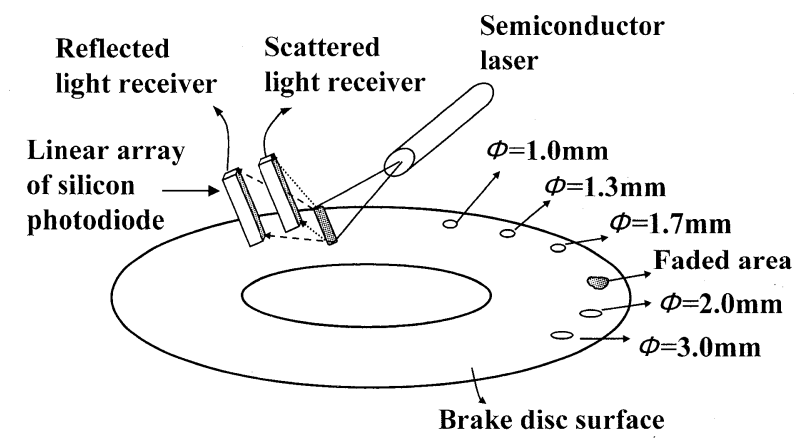

Fig.2 Samples of the hole and fade on the brake disc surface.

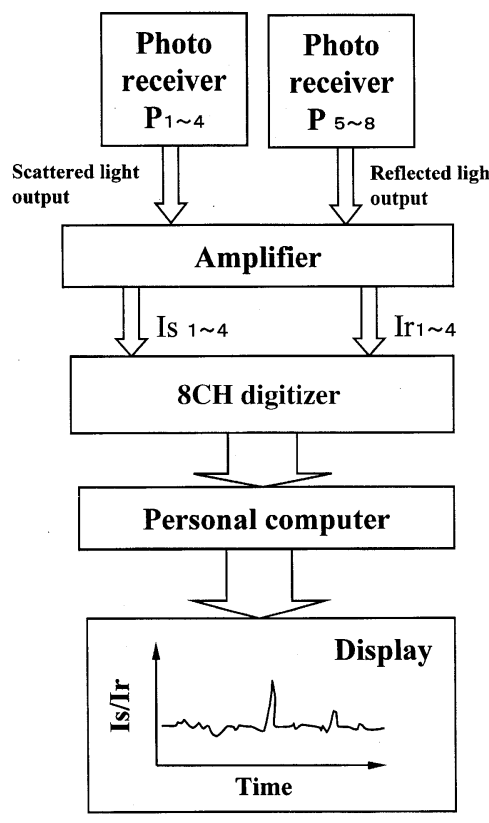

Fig.3 Data processing system.

\section{Experimental Results and Discussion}

The preliminary experiment was carried out using drilled hole as a flaw in place of practical holes. Figure 4 shows an example of both the received light intensity and the ratio of scattered light to reflected light when the surface of the brake disc was scanned by the sheet-like laser light; (a) the scattered light intensity $I_{\mathrm{s}}$, (b) the reflected light intensity $I_{\mathrm{r}}$ and (c) the ratio of both light intensities $I_{\mathrm{S}} / I_{\mathrm{r}}$. It was shown that the holes increase the scattered light intensity and at the same time decrease the reflected light intensity. This is just the predicted aspect shown in Fig.1 (b). The flaws, therefore, can be detected by only $I_{\mathrm{S}}$ or $I_{\mathrm{r}}$.

The $I_{\mathrm{S}} / I_{\mathrm{r}}$ ratio, however, will be more enlarged at the flaws than only $I_{\mathrm{S}}$ or $I_{\mathrm{r}}$ and will have a higher sensitivity, i.e., a higher signal to noise ratio, $S$. The signal to noise ratio will be $S=S_{\mathrm{s}} \times$ $S_{\mathrm{r}}$, where $S_{\mathrm{s}}$ is the signal to noise ratio for the scattered light intensity and $S_{\mathrm{r}}$ for the reflected light intensity. For example, it can be determined by Figs.4 (a), (b), and (c) that $S_{\mathrm{s}}, S_{\mathrm{r}}$, and $S$ are about $S_{\mathrm{s}}=5, S_{\mathrm{r}}=3$, and $S=15$ for a hole diameter of $3.0 \mathrm{~mm}$. This is the main reason to use the ratio, $I_{\mathrm{S}} I_{\mathrm{r}}$, in place of only $I_{\mathrm{S}}$ or $I_{\mathrm{r}}$ for the flaw detection. Furthermore, it can be seen that a hole with a diameter of $\Phi=1.3 \mathrm{~mm}$ can not be detected by only the scattered light intensity (Fig.4 (a)), and a hole of $\Phi=1.0 \mathrm{~mm}$ can not be detected by only the reflected light intensity (Fig.4 (b)). But both holes can be detected by using the ratio of the scattered to reflected light intensity (Fig.4 (c)).

The color change due to cutting or grinding of the brake disc, in this case the black marked point on the surface, decreases both scattered and reflected light intensities at the same time and therefore has a small effect on $I_{\mathrm{S}} I_{\mathrm{r}}$. This is another reason to use $I_{\mathrm{S}} / I_{\mathrm{r}}$ in place of only $I_{\mathrm{S}}$ or $I_{\mathrm{r}}$. A CCD camera was also used for the flaw detection, but, the camera could not distinguish the flaw from the black marked point.

The detection sensitivity of this method depends on the ratio of the flaw area to the cross-sectional dimension of the sheetlike laser light. The length of the cross section, $l$, determines the width of the scanning, and the width $w$ mainly determines the

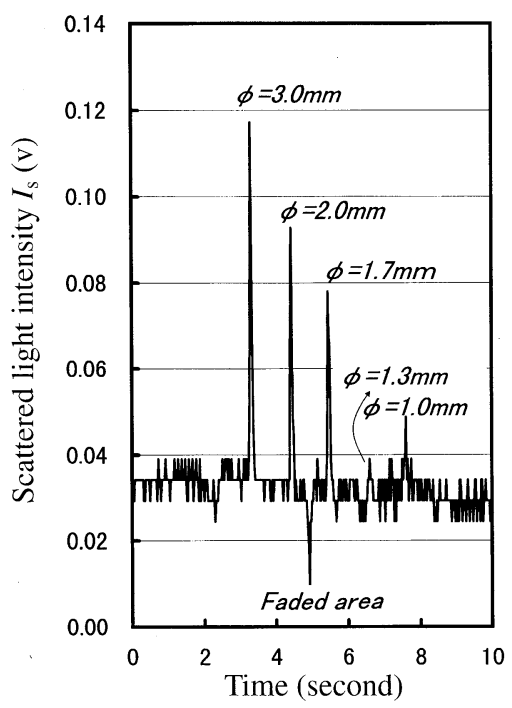

(a)

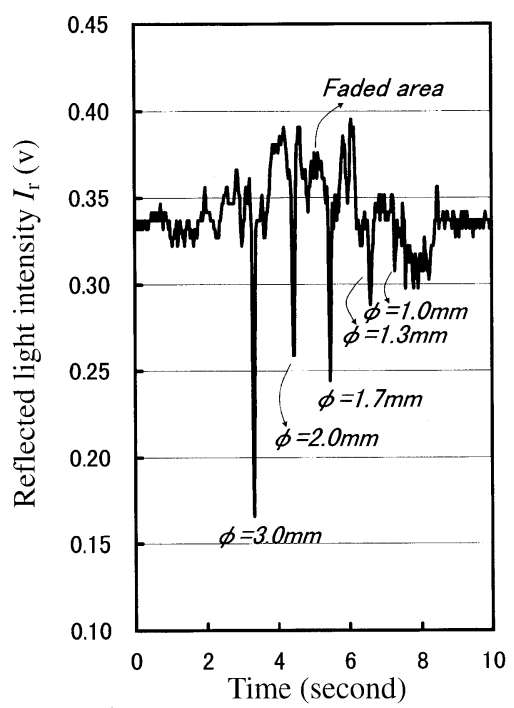

(b)

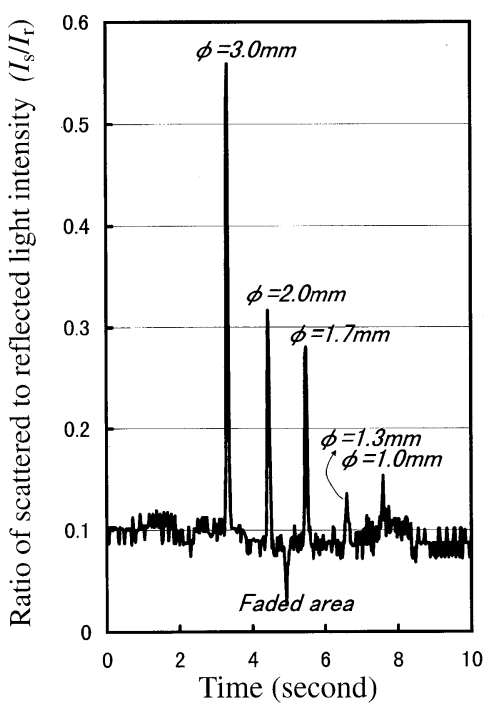

(c)

Fig.4 Scattered and reflected light intensities and the ratio between them at disc radius $R=100 \mathrm{~mm}$ on the brake disc surface with some holes and a fade and the ratio of them. These were obtained with a data aquisition sampling frequency of $80 \mathrm{~Hz}, \mathrm{a}$ sheet like laser beam of length, $l=10 \mathrm{~mm}$, and width, $w=0.5 \mathrm{~mm}$. (a) Scattered light intensity, (b) Reflected light intensity, and (c) Ratio of scattered to reflected light intensity. 
diameter of the detectable smallest hole, $\Phi_{\mathrm{m}}$. The high sensitivity will, therefore, be obtained for small $l$ and $w$ and large $\Phi_{\mathrm{m}}$. Generally, it will be sufficient to be smaller than the hole diameter, but a long $l$ will be desirable from a practical point of view. For valid detection of the flaw, it is necessary to find the metting point $i$ and $\Phi_{\mathrm{m}}$. The long $l$ can be obtained since the smallest diameter is usually given. It was found from the preliminary experiment that the long $l$ is about $10 \mathrm{~mm}$ for $\Phi_{\mathrm{m}}=2 \mathrm{~mm}$.

It is also necessary for valid detection of the flaw to acquire data for the light intensity just on the flaw. The minimum sampling frequency of data acquisition, $f_{\mathrm{m}}$, can then be calculated by the radius of the brake disc, R, the hole diameter, $\Phi_{\mathrm{m}}$, and the detection speed, i.e., the speed of the disc revolution, $\omega$, as follows.

$$
f_{\mathrm{m}}=2 R \omega / \Phi_{\mathrm{m}}, \quad \omega=2 \pi / T,
$$

where $T$ is the revolution time, i.e., the required detection time. As an example, we obtain the minimum sampling frequency, $f_{\mathrm{m}}$ $=628 \mathrm{~Hz}$, for $T=1 \mathrm{~s}, R=100 \mathrm{~mm}$ and $\Phi_{\mathrm{m}}=2 \mathrm{~mm}$.

Two practical methods will be considered for the sensor head. One consists of some pair of sensors including a light source and a light receiver, scanning all over the surface. For example, a brake disc of radius $R=80 \mathrm{~mm}$ can be inspected by 8 pairs of sensors when we use a probing sheet light of length $l=10 \mathrm{~mm}$. In this case a 16 channel $\mathrm{A} / \mathrm{D}$ converter will be required. The other method consists of a pair of sensors with a moving mechanism to scan all over the surface.

\section{Conclusion}

An optical system applicable for practical use has been developed for detecting the surface flaws of a molding. The system can detect flaws on a brake disc of radius $100 \mathrm{~mm}$ within a few seconds by using a data aquisition frequency of $1 \mathrm{kHz}$ and a probing light width of $10 \mathrm{~mm}$.

\section{Acknowledgement}

Technical support throughout the present study was provided by Mr T. Inaki of Fukui University, Fukui, Japan.

\section{References}

1) M. Ueda, M. Kawazu, and H. Suga: Opt. Lasers Eng. 28 (1997) 71.

2) T. Yamamoto, R. Makino, and M. Ueda: J. Jpn. Soc. Tech. Education 39-4 (1997) 231.

3) J. Chen, Y. E. Lee, K. Taniguchi, K. Asada, and M. Ueda: Rev. Laser Eng. 26 (1998) 176. 